\title{
DATA-DRIVEN SONIFICATION OF CFD ANEURYSM MODELS
}

\author{
Daniel E. MacDonald \\ University of Toronto \\ Biomedical Simulation Laboratory \\ Toronto, Canada \\ demacdo@mie.utoronto.ca
}

\author{
Thangam Natarajan \\ University of Toronto \\ Biomedical Simulation Laboratory \\ Toronto, Canada \\ thangam@mie.utoronto.ca
}

\author{
Richard C. Windeyer \\ University of Toronto \\ Centre for Drama, Theatre, \\ and Performance Studies \\ Toronto, Canada \\ rwindeyer@me.com
}

\author{
Peter Coppin \\ OCAD University \\ Perceptual Artifacts Lab \\ Toronto, Canada \\ pcoppin@faculty.ocadu.ca
}

\author{
David A. Steinman \\ University of Toronto \\ Biomedical Simulation Laboratory \\ Toronto, Canada \\ steinman@mie.utoronto.ca
}

\begin{abstract}
A novel method is presented for inspecting and characterizing turbulent-like hemodynamic structures in intracranial cerebral aneurysms by sonification of data generated using Computational Fluid Dynamics (CFD). The intention of the current research is to intuitively communicate flow complexity by augmenting conventional flow visualizations with data-driven sound, thereby increasing the ease of interpretation of dense spatiotemporal data through multimodal presentation. The described implementation allows the user to listen to flow fluctuations thought to indicate turbulent-like blood flow patterns that are often visually difficult to discriminate in conventional flow visualizations.
\end{abstract}

\section{INTRODUCTION}

\subsection{Aneurysms and sounds}

A cerebral aneurysm is the localized bulging of a weakened artery wall within the brain. Often, no symptoms of the aneurysm are present in unruptured cerebral aneurysms. An estimated 2-5\% of adults harbour an aneurysm, of which the risk of rupture per year is approximately $1 \%$. Of ruptured aneurysms, $40 \%$ are fatal [1]. Rupture occurs when the stresses exerted on the artery wall exceed the strength of the wall.

Blood flow patterns (hemodynamics) are thought to contribute to the initiation, growth, and rupture of intracranial aneurysms [2], by stimulating degenerative remodelling (weakening) of the artery wall. Because the forces associated with these flows are difficult to measure non-invasively using current technologies, medical image-based CFD has been used to investigate these hemodynamic forces retroactively $[2,3,4]$. Using digitized patient-specific vasculature models extracted using 3D angiography techniques, blood flow is simulated using advanced numerical methods. The results of such simulations are typically visualized as part of the process of data analysis.

These previous investigations have revealed turbulent-like flow instabilities within aneurysms or the vessels that host them,

\section{(c) (i) (8)}

This work is licensed under Creative Commons Attribution Non Commercial 4.0 International License. The full terms of the License are available at http://creativecommons.org/licenses/by-nc/4.0

This research was funded by the Canadian Natural Sciences and Engineering Research Council, the Social Sciences and Humanities Research Council, and the Heart \& Stroke Foundation. with implications for aneurysm rupture and initiation [2,3]. Such instabilities are typically represented through conventional visualization paradigms (glyphs, streamlines, isosurfaces), and though these can provide tangible and intuitive representations of the hemodynamics, visual differences among unstable laminar, transitional, and turbulent flow regimes in complex vascular geometries are often indiscernible. In this context, a novel method originally inspired by Doppler ultrasound auscultation is presented for communicating the nature of the flow by augmenting conventional flow visualizations with data-driven sound (sonification).

Auscultation refers to the established medical technique of listening to the sounds of the body for evaluation purposes [5]. For example, clinicians are trained to identify turbulent blood flows by recognizing auditory properties of duration, quality, and timing during examination of bruits in the carotid artery [5]. Doppler auscultation has been used in preliminary stages to listen to valvular movements and blood flow velocity sounds during cardiac examination, resolving sounds undetected by stethoscope [6], though to our knowledge, this technique has not been demonstrated on intracranial aneurysms.

Intracranial saccular aneurysms have been observed to emit acoustic signals (bruits) as a result of fluctuating hemodynamic flows thought to be characterized as turbulent-like [7, 8, 9]. These vibrations are difficult to measure in vivo due to difficulty differentiating these bruits from other bodily sounds, and as such are not a reliable source of diagnostic information [8].

Inspired by these techniques, we present a method for sonifying numerically simulated flow of intracranial aneurysms for the purposes of identifying hemodynamic flow complexity during the process of flow visualization.

\subsection{Conventional visualization of CFD data}

Standard visualization techniques for representing multidimensional CFD datasets include static or animated views of isosurface rendering, glyphs, or pathline and streamline representations. Fig. 1 shows these conventional representations applied to our intracranial aneurysm CFD data set.

Particle pathline animations from CFD data provides a tangible representation of fluid flow and are often in good agreement with our intuitive understanding of structure and movement. Due to the large number of particles required to represent the spatiotemporal flow, the visual difference between laminar, transitional, and turbulent flow is difficult to discern - all may look chaotic, but each 


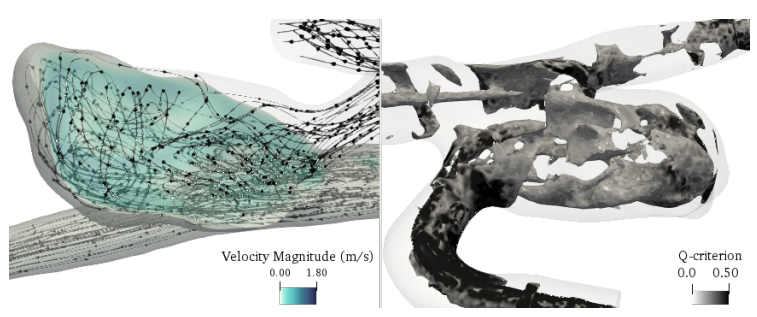

Figure 1: Examples of conventional flow visualizations created using ParaView. On the left, streamlines of velocity are augmented by glpyhs, with coloured slice indicating internal flow velocity of the aneurysm sac. On the right, surfaces of Q-criterion indicate regions of unstable flow.

behaves in accordance with differing principles, and may have distinct consequences for artery wall remodelling.

Isosurfaces and thresholds of computed quantities, such as velocity, provide an uncluttered representation resembling discrete visual blobs, though such blobs may obscure or occlude regions of data. These visualizations may be more visually abstract than pathlines and are subject to further sculpting by the user. Isosurfaces of Q-criterion, a representation of vortex cores, are particularly useful for assessing location and intensity of turbulent-like flow [10]. There is nothing inherently visual about our CFD derived data - seed particles, surfaces, thresholds, and colours are ascribed by convention and experience. Conceptually, temporal fluctuations are also suitable for listening, and as mentioned previously, aneurysms are known to emit bruits as a result of velocity and pressure instabilities. From results of CFD, velocity or pressure measurements for each and every spatial location can be easily written as a WAV file for playback, however this method lacks control and ease of further manipulation.

Visually communicating spatiotemporal instabilities ignores the existing strengths of the human auditory system for resolving frequencies as compared to visual perception of spatial frequencies. For example, attempting to visually distinguish a difference of pitch between vibrating strings ignores the auditory ability to distinguish subtle pitch differences, of which Moore indicates can be as small as $0.2 \%$ [11].

This lack of visual acuity is not only a problem of aneurysm visualization, but flow visualization in general. By Nyquist's theorem, in order to produce a faithful digital reproduction of any signal, the sample rate of the digital medium (audio or video file) must be at least twice that of the highest frequency component of interest contained within the original signal. For example, given a video frame rate of $60 \mathrm{~Hz}$, a fluctuating signal of at most 30 $\mathrm{Hz}$ can be reproduced. As the frequency of the fluctuating signal increases, a higher video frame rate would be required. Given the frequency range of interest for bruits observed in intracranial aneurysms (200-800 Hz) [8], uncommonly high frame rates (400$1600 \mathrm{~Hz}$ ) or greatly slowed video would be required to communicate such instabilities, without even considering whether we may visually perceive these fluctuations.

\subsection{Sonification of CFD Data}

Sonification techniques can be broadly separated into five categories: audification, auditory icons, earcons, parameter mapping, and model-based sonification. Of these techniques, parameter mapping (the mapping of data dimensions to auditory dimensions) is the most common technique [12], allowing continuous communication of information and greater flexibility of design compared to audification, auditory icons, and earcons. Mapping relationships must be considered very carefully in order to generate an informative sonification.

The generation of a parameter mapping sonification representation consists of three stages: choice of the mapping strategy (ie. which data dimension maps to which auditory dimension), choice of polarity, and psychophysical scaling [13]. Within the choice of mapping strategy, the choice of data scale on which to operate (globally, or within local subsections) must also be considered with great care.

Several works have addressed the sonification of spatiotemporal flow data. Klein and Staadt [14] describe an implementation using wind sounds to indicate the velocity and vorticity at a local point within a three dimensional rectilinear vector field. By sampling a local volume representing the users headspace, vector properties manipulate a static sound sample using head-related transfer functions (HRTFs) to convey vector direction, magnitude, and vorticity to the user. Stereo speaker setups as well as headphones with head tracking systems were tested. The authors emphasize the need for an appropriately sized sample volume (headspace) for evaluating vorticity; too small, and the vectors are too similar, yielding low vorticity, while too high, and the sonification will always sound cacophonous.

Kasakevich et al [15] describe a multi-modal interface for use in a computational steering environment to augment 3D visualization using sonification for the purposes of interpreting velocity values and localizing vortex cores. Conceptually, it is similar to the work of Klein and Staadt, though this implementation is performed on an unstructured grid with a variable listening radius. Importantly, a usability analysis was presented along with the implementation. Multi-modal feedback was shown to improve localization of vortex centers when compared to visual-only feedback. Audiovisual feedback provided the greatest performance, followed by visual-only, with poor performance using audio-only feedback. Participants took less time and used shorter path lengths when using audio-visual feedback, though accuracy was unaffected. In evaluating polarity of auditory feedback in the audio-visual tests, accuracy improved for positive amplitude conditions, though path length remained unaffected.

Menelas [16] provides a technique for interactively assessing open-cavity flow phenomena using virtual reality (VR) techniques. With an emphasis on visual feedback, Menelas attempts to address the complexity related to the analysis of CFD data using visual, haptic, and audio feedback. User feedback was provided from four participants, two users versed in CFD and two computer scientists, though no feedback is relevant to the auditory domain.

Although the current research began independently of the work of Klein and Staadt, Kasakevich, and Menelas [14, 15, 16], it has converged upon a similar solution in which high-density data sonification is navigated within a three-dimensional auditory field with a focus on the communication of spatiotemporal data. The design of the current work benefited from Klein and Staadts observations regarding sample volume sizing; a balance must be struck between providing a granular representation of a single point or a cacophonous representation of a large volume.

The motivation for the current work was to address the shortcomings of data visualization with respect to temporal fluctuations, whereas previous works sought to address the visual clutter associated with high-dimensional datasets. The works of Klein and 
Staadt, Kasakevich, and Menelas directly communicate time domain values, such as velocity of flow, in order to reduce the strain on visual bandwidth by mapping magnitude of vectors directly to amplitude or filter cutoff $[14,15,16]$. For those previous works, this is suitable, allowing the user to directly query the data and receive auditory feedback proportional to the measured data. Rather than communicating vector magnitude, the current research seeks to sonify the temporal evolution of the vector, particularly the complexity of instability through time. For example, a region of high flow velocity that is stable in time may indicate a region of low complexity, while a slow-moving but unsteady flow may indicate a region of high complexity.

The current work seeks to decompose the time domain values (velocity) in the frequency domain in order to discriminate regions of unstable flow. The current method allows inspection of individual frequency bands independently. Although point vector values remain the basis for our current work, the frequency domain processing between these vector values and the audio output distinguishes the current research from previous works. By sonifying the spectrogram, the envelope shape (percussive or smooth nature) is communicated along with spectral content.

Additionally, the flow is pulsatile, indicating that greater consideration should be given to the evolution of the signal through each period, i.e., cardiac cycle. The temporal resolution of the data is therefore considered very important in order to perform meaningful manipulations in the frequency domain, and as such is finer than previous works. In combination with use of a fine, unstructured mesh, our datasets tend to be large and unwieldy, with number of points on the order of hundreds of thousands or millions, and temporal resolutions on the order of milliseconds.

\section{METHOD}

High-fidelity CFD data sets produced using cases from the opensource Aneurisk database [17] serve as the basis of this methodology. Within an interactive visualization environment (ParaView an opensource scientific visualization tool [18]), the average power spectral density (PSD) of velocity-time traces is computed for a probed sample volume, defined by centre point and radius, in order to evaluate a local region of flow within the aneurysm sac. The probe radius is chosen based on two considerations: first, the real-time computation must support interactivity, and second, the probe should not be too spatially localized or too global. To define this balance of local and global volume, the probe diameter should not exceed the neck diameter of the aneurysm and should be small enough to separate coherent Q-criterion structures. The frequency content and time-varying power of the resulting spectrogram are communicated to a real-time audio server (SuperCollider sound server [19]) to control the frequency and loudness of a bank of audio-rate sound generators. The summation of these generators results in a harmonically rich signal representative of the probed location.

This on-the-fly implementation is contained (though not constrained) within the Python shell and intended to run alongside a synchronized animation. Sound networking protocol Open Sound Control (OSC) is used to communicate the spectrogram data between the live ParaView environment and audio server. The audio presentation of the signal is highly configurable while maintaining audio-rate synthesis, allowing the user to pitch, slow down, or threshold by frequency band in real-time, synchronized to the visualization.

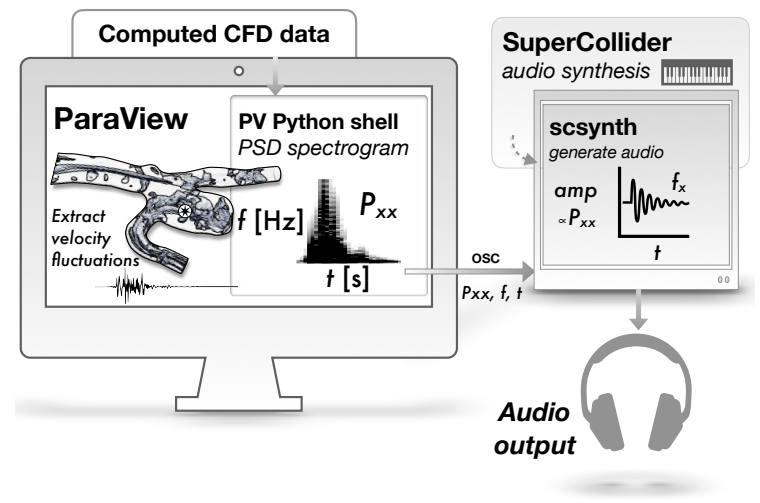

Figure 2: Overview of the real-time sonification using ParaView and SuperCollider.

\subsection{Mapping strategy, polarity, and psychological scaling}

Of the various sonification techniques, the flexibility and continuous nature of parameter mapping was most influential in design. The structure of the instrument was designed such that any aneurysm dataset (or any collection of spatiotemporal data) may be used as input with little manipulation (only requiring a transpose of velocity vectors). As the user probes the data, varying audio outputs are generated local to the probe. As such, different frequency content and amplitude envelopes are readily generated for each new sample of the dataset.

A guiding philosophy for sonic aesthetics was to caricature the existing physiological phenomena by generating sound inspired by fluid sounds existing in nature, and not necessarily to recreate the full acoustic field tied to the flow. By taking advantage of our intuitive understanding of how certain sounds (wind, bubbles) map to velocity of fluid, the cognitive load is reduced. The basis of our parameter mapping was generated as simply as possible: the spectral content of velocity is mapped with auditory frequency values, while magnitude of spectral content is mapped with loudness. The parameters to be mapped are all derived from physical velocity values, and as such are not subject to ambiguity of order; the mapping is intended to resemble a simulation of underlying physical phenomena. As such, polarity choices were made to follow the logic of ecological perceptions. Use of spatial audio was considered to represent space of the mesh, but was ultimately found more useful for communicating the kinetic directionality of flow within the sample volume.

\subsection{Implementation}

Fig. 2 provides an overview of the method of sonification. In the following sections, the following aspects of the real-time method of sonification are described in detail:

- Probing the local volume for extraction of point velocity data

- Filtering velocity data by spectral power index (SPI) criteria

- Computing the PSD spectrogram from the filtered velocity data

- Passing the PSD data to SuperCollider to produce audio 


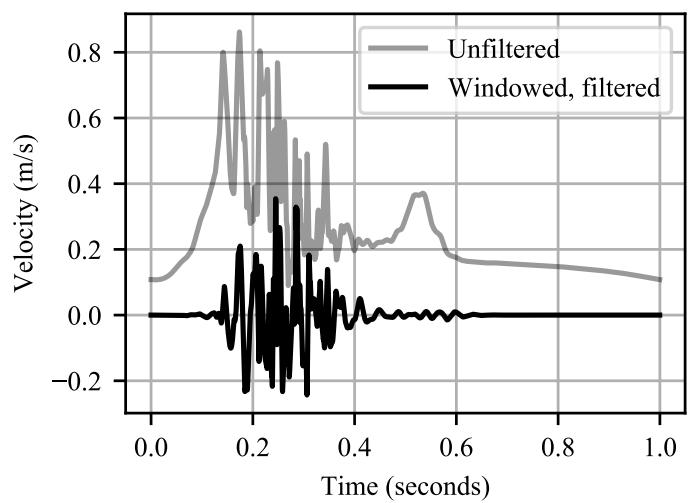

Figure 3: Isolation of velocity fluctuations by high-pass filtering the pulsatile signal according to SPI criteria.

Data is stored in binary data frames and accessed interactively from the Paraview render view. SuperCollider is used to generate the sonification from the flow data.

\subsection{Probing Data}

Much like a stethoscope for the body, the user may interactively drag the probe (representing the sample volume) through the domain to listen to the local frequency spectrum. The chosen probe radius used is $0.25 \mathrm{~mm}$, containing approximately 50 discrete points within the volume. A list of point identifiers is generated for a 20 uniformly distributed random points within this local volume. The velocity values for these points through time will serve as the basis for the sonification, to be discussed in subsequent sections. This sonification is intended to accompany a synchronized visualization with time duration chosen by the user. As such, this pipeline is updated once for each period of flow. The data has temporal resolution of $0.004 \mathrm{~s}$ (2500 samples/second) and about 500 000 points.

\subsection{Filtering Data}

As shown in the Fig.3, the velocity content for each point is extracted and filtered by criteria of spectral power index (SPI) to isolate flow instabilities.

SPI is a frequency domain decomposition method introduced by Khan et al [20] as a means to quantifying the transient instabilities of turbulent-like flows without cycle-to-cycle variations. This decomposition separates the pulsatile carrier signal from the turbulent-like flow instabilities. By using a high pass filter to remove the $99 \%$ most energy-carrying frequencies (corresponding to a cutoff frequency of $25 \mathrm{~Hz}$ ), flow instabilities can be isolated. SPI is defined as the ratio of energy contained within the flow instabilities as a fraction of all signal energy.

\subsection{Computing the average spectrogram}

The local PSD spectrogram for each point is calculated using a window length of 256 samples with 192 sample overlap, resulting in a smooth, temporally shifting view of the power content for each frequency bin. The average spectrogram for the sample volume is

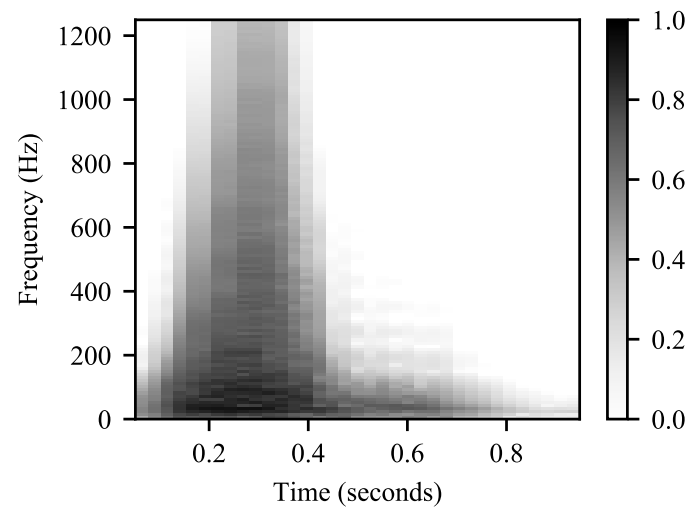

Figure 4: Average power spectral density computed for $\mathrm{N}=20$ sample points in radius $0.25 \mathrm{~mm}$.

then computed (Fig.4). This matrix of power content corresponds to 128 frequency bins $\mathrm{x} 36$ time bins.

\subsection{Generation of sonification}

A synthesizer template used for creating synth nodes (synthdef) was generated in the SuperCollider environment and stored on scsynth (a real-time SuperCollider audio server [19]). Using sound networking protocol Open Sound Control (OSC), the power matrix is sent to scsynth.

The frequency content and time-varying power of the computed spectrogram are fed to a bank of sound generators. Each frequency bin of the spectrogram corresponds to the frequency of a generator. The time-varying power values of the spectrogram are used to modulate the loudness (amplitude) of each generator in time.

An array of synth nodes are generated, with each node procedurally assigned a range of frequency bins from the power matrix. The power matrix is broken into chunks by frequency, with each chunk containing five frequency bins to be generated per node. The power associated with each bin corresponds to the temporal loudness (amplitude envelope) of the sound as well as a bubble parameter, while the frequency value corresponds to the resonant frequency of a constant skirt gain filter (Ringz generator) with noise input. The bubble parameter controls the cutoff frequency and modulation depth of a resonant high-pass filter. By pseudorandomly shifting the cutoff frequency of the high pass filter near the resonant frequency of the Ringz generator, and with a shift depth proportional to the temporal power, sound textures resembling bubbles are generated to indicate a higher level of flow complexity.

The summation of these generators results in a harmonically rich signal with frequency content varying in time. By tweaking the bubble parameter, the output sound varies between a harmonically clean sound reminiscent of Doppler auscultation, to windy sound textures, and more evocative ecological fluid flow sounds. By modulating this parameter with the amplitude envelope, the resulting signal sounds more chaotic during the systolic pulse of flow.

The apparent directionality of flow is communicated by rotating an ambisonic B-format sound field around the user. Given 
the position of the aneurysm model onscreen, the directionality of flow relative to the user is determined and used to spatialize the sound; for flow visually moving left-to-right, the perceived sound field rotates clockwise around the user, and vice versa. The perceived rotation angle indicates the relative velocity orthogonal to the user.

\subsection{User Interaction}

The signal can be pitched up (maintaining relational frequency content) and slowed down while maintaining audio-rate synthesis, and can be pruned of low-amplitude frequency content or filtered in order to evaluate content by frequency band.

This on-the-fly generation is intended to accompany a conventional flow visualization in ParaView. All computed data (signal filtering and extraction of PSD) is computed within the Paraview python shell.

\subsection{Plan for User Study}

A preliminary user study is planned to test the accuracy and speed of discriminating a variety of complex flows, compared to using standard visualizations alone.

Conventional visualizations of intracranial aneurysms effectively communicate flow pattern, but do not effectively communicate flow quality (laminar, transitional, turbulent). By allowing users to discriminate the spectral quality of the flow by augmenting the visualization with sound, it is hypothesized that users will be able to better identify turbulent flow as compared to standard visualization alone.

Two training steps will be necessary. First, the correspondence between sonified spectral data and turbulent-like flow will be established in agreement with current auscultation literature, noting timings, pitch, and intensity [5]. Second, flow quality will be demonstrated using conventional flow visualizations, particularly pathline representations.

Three hypothesis have been generated regarding interpretation of unstable flow in numerically simulated aneurysms:

1. Users can accurately discriminate levels of turbulence through sound alone.

2. Users cannot accurately discriminate regions of turbulence by visuals alone.

3. Users will be able to best identify regions of turbulence using multimodal representation.

First, users will be be asked to comparatively listen to sounds alone and rank which contains higher complexity or turbulence. User responses will be compared to known quantities of flow complexity. Second, the users will be asked to visually compare and rank-order regions of complex flow in a conventional visualization. Third, a conventional visualization will be shown to the user with sonified data for preselected regions. The user will be asked to identify which region contains more turbulence. Evaluation of the user study will occur in April-May 2018.

\section{RESULTS AND DISCUSSION}

An current example of the implemented method can be found at [21].

The present framework of sonification results in a highly configurable audio representation allowing on-the-fly exploration of flow instabilities within CFD data. Frequency bands can be individually emphasized or extracted for closer inspection. Different sound textures, ranging from Doppler-inspired sounds to bubbly flow sounds, exist within the instrument framework. This sonification runs in real time next to a simplified conventional visualization on consumer grade hardware, allowing for flexible inspection of data.

Careful choice of mapping strategy, parameter polarity, and psychological scaling provide a range of sonic palettes for known conditions of flow within intracranial aneurysms. The prototype has been tested with five aneurysm datasets, each of similar temporal and spatial resolution but varying flow complexity. Further exposure to new datasets will confirm the existing method of psychological scaling, or suggest further refinement to accommodate a broader range of flow conditions.

The biological and clinical implications of high frequency content from a decomposed velocity signal are not the basis of this report; these effects are discussed in greater depth in CFD literature. The physiological presence of high frequency fluctuations, along with frequency-specific amplitude envelopes, are to be communicated to the user for interpretation. Other simplified strategies such as directly communicating Q-criterion to indicate turbulent flow were sonified, but by reducing the number of communicated parameters, the feedback system was found too simplistic for anything other than locating regions of instability. If it were as easy as presenting a prepared quantity, sonification would not be necessary; by design, this sonification attempts to maintain the nuance of the original signal for interpretation by a trained user. The objective of the current research is to present the spectral data using audio, and not to interpret the data.

The frequency resolution of the calculated PSD is limited by the temporal resolution of the data. By windowing the data, variation in time is gained at the expense of frequency resolution. This is a reasonable balance if the signals often approximate noise; broad temporal and spectral characteristics are communicated. From this balance, there are two potential disadvantages. First, the hard-set designation of frequency bands results in similar timbral properties between datasets. Secondly, and perhaps more importantly, if the signals have strong harmonic content, greater frequency resolution may be required to explore spectral relationships. Given the existing knowledge of spectral content in turbulent-like flow, the current chosen windowing parameters are acceptable.

By choosing the data for sonification based on known physiological parameters and carefully scaling, initial impressions appear to indicate good correspondence between data and sonification.

The methodological framework of the current research has been presented without formal user-study, and as such further evaluation of the design is the most crucial next step. In addition to evaluation of the hypotheses described in section 2.8 , the effectiveness of the multidimensionality presented within the framework should be evaluated. From preliminary observation of the prototype, there is a possibility that the salience of each dimension within the sonification may not be perceptually balanced. For example, temporal characteristics of the sound may overshadow the harmonic content of the signal, which may indicate a need for finer resolution in harmonic content in order to discriminate different flows. Similarly, fluctuations persisting long in into the cardiac cycle may be perceived as more turbulent compared to short, intense bursts. Such an assessment may indicate that temporal envelope characteristics may require further refinement. Until the 
framework is evaluated through user training and user study, the effectiveness of this sonification can by only incompletely assessed based on the design criteria of the authors.

A fundamental design limitation of the present framework lies in the ill-defined relationship between hemodynamic forces and degenerative remodelling of the aneurysm wall [2]. As such, harmonic and temporal components of the decomposed flow data are treated approximately equally to retain characteristics of the physical phenomena.

This work extends previous sonifications of multidimensional vector data by directly addressing the shortcomings of data visualization with respect to temporal instability. This insight resulted from in-depth knowledge of the physical and simulated hemodynamics specific to the current application of intracranial aneurysms.

The proposed method for interpreting cerebral flows is not limited by application, and may be useful for other time-series spatial datasets containing instabilities. A similar implementation has also been tested in the 3D creation suite Blender [22], and would presumably function in any other python-based environment.

\section{CONCLUSIONS}

A novel method for sonifying CFD data for the purpose of better communicating high frequency fluctuations within simulated intracranial aneurysmal flows is described. This frequency-domain method is intended to augment conventional visualization in order to ease the interpretation of dense spatiotemporal datasets by allowing the user to visually concentrate on one field while listening to another. This method for data inspection builds on established analysis of intracranial hemodynamics with a focus on real-time inspection inspired by the existing field of auscultation.

\section{REFERENCES}

[1] "Brain aneurysm statistics and facts," Web Site, 2018. [Online]. Available: https://www.bafound. org/about-brain-aneurysms/brain-aneurysm-basics/ brain-aneurysm-statistics-and-facts/

[2] K. Valen-Sendstad, K. A. Mardal, and D. A. Steinman, "High-resolution CFD detects high-frequency velocity fluctuations in bifurcation, but not sidewall, aneurysms," Journal of Biomechanics, vol. 46, no. 2, pp. 402-407, 2013.

[3] K. Valen-Sendstad, M. Piccinelli, and D. A. Steinman, "High-resolution computational fluid dynamics detects flow instabilities in the carotid siphon: Implications for aneurysm initiation and rupture?" Journal of Biomechanics, vol. 47, no. 12 , pp. 3210-3216, 2014.

[4] N. Varble, J. Xiang, N. Lin, E. Levy, and H. Meng, "Flow Instability Detected by High-Resolution Computational Fluid Dynamics in Fifty-Six Middle Cerebral Artery Aneurysms," Journal of Biomechanical Engineering, no. 6, p. 061009.

[5] A. J. Taylor, Learning Cardiac Auscultation: From Essentials to Expert Clinical Interpretation. Springer.

[6] M. J. Mc Loughlin and S. Mc Loughlin, "Cardiac auscultation: Preliminary findings of a pilot study using continuous Wave Doppler and comparison with classic auscultation," International Journal of Cardiology, no. 2, pp. 590-591.
[7] L. N. Sekhar, M. Sun, D. Bonaddio, and R. J. Sclabassi, "Acoustic recordings from experimental saccular aneurysms in dogs." Stroke, no. 8, pp. 1215-21.

[8] G. G. Ferguson, "Turbulence in human intracranial saccular aneurysms." Journal of neurosurgery, no. 5, pp. 485-97.

[9] Y. Kurokawa, S. Abiko, and K. Watanabe, "Noninvasive Detection of Intracranial Vascular-Lesions By Recording Blood-Flow Sounds," Stroke, vol. 25, no. 2, pp. 397-402, 1994.

[10] G. Haller, "An objective definition of a vortex," Journal of Fluid Mechanics, vol. 525, pp. 1-26, 2005.

[11] B. C. J. Moore, "Frequency difference limens for short duration tones," The Journal of the Acoustical Society of America, no. 3, pp. 610-619.

[12] G. Dubus and R. Bresin, "A systematic review of mapping strategies for the sonification of physical quantities," PLoS $O N E$, vol. 8, no. 12, 2013.

[13] T. Hermann, A. Hunt, and J. G. Neuhoff, The Sonification Handbook, 2011.

[14] E. Klein and O. G. Staadt, "Sonification of three-dimensional vector fields," in Proceedings of the 12th SCS High Performance Computing Symposium (HPC 2004), part of the SCS Advanced Simulation Technologies Conference (ASTC'04), 2004.

[15] M. Kasakevich, P. Boulanger, W. F. Bischof, and M. Garcia, "Augmentation of Visualisation Using Sonification : A Case Study in Computational Fluid Dynamics," IPT-EGVE Symposium, 2007.

[16] B.-A. J. Menelas, "Interactive analysis of cavity-flows in a virtual environment," Proceedings of the 28th Spring Conference on Computer Graphics, pp. 31-38, 2012.

[17] Aneurisk-Team, "AneuriskWeb project website, http://ecm2.mathcs.emory.edu/aneuriskweb," Web Site, 2012. [Online]. Available: http://ecm2.mathcs.emory.edu/ aneuriskweb

[18] U. Ayachit, "The ParaView Guide: A Parallel Visualization Application," Kitware.

[19] J. McCartney, SuperCollider, 2018. [Online]. Available: https://supercollider.github.io

[20] M. O. Khan, C. Chnafa, D. Gallo, F. Molinari, U. Morbiducci, D. A. Steinman, and K. Valen-Sendstad, "On the quantification and visualization of transient periodic instabilities in pulsatile flows," Journal of Biomechanics, vol. 52, pp. 179-182, 2017.

[21] D. MacDonald, Sonification of flow instabilities in intracranial aneurysms, Biomedical Simulation Laboratory, University of Toronto, 2018. [Online]. Available: https://www.youtube.com/watch?v=UmDvnPjnpV4

[22] Blender Online Community, Blender - a $3 D$ modelling and rendering package, Blender Foundation, Blender Institute, Amsterdam, 2017. [Online]. Available: http: //www.blender.org 